\title{
Metamemory-Memory Behavior Relationships in Young Children: Evidence from a Memory-for-Location Task
}

\author{
WolfGang Schneider \\ Max Planck Institute for Psychological Research. Munich
}

AND

Beate Sodian

University of Munich

\begin{abstract}
Relationships among metamemory. memory behavior, and memory performance in 4- to 6-year-old children were studied in two experiments, employing a memoryfor-location task in which retrieval cues were available as memory aids. There were substantial correlations among task-related metamemory. memory behavior (i.e.. use of retrieval cues). and memory performance even in 4-year-olds. Very few children used retrieval cues without being aware of at least some of their functions. Cue use. cue effectiveness. and metamemory concerning retrieval cues were shown to increase over the preschool years. One of the most interesting developmental trends seemed to be the increasing ability to establish relationships between cues and targets even if these are not highly associated. These results are discussed with regard to previous research on young children's use and understanding of retrieval cues. $s$ is Academic Press. Inc
\end{abstract}

Research on metamemory development in young children has shown that a basic idea about the nature of memorization is acquired in the early preschool years. Some of the variables which affect performance on memory tasks, for instance, study time, age, and amount of items to be remembered, are understood even before the age of four years (Wellman, 1977) and some simple strategies to enhance prospective retrieval are well-known to kindergartners (Kreutzer, Leonard, \& Flavell, 1975).

Young children's behavior in memory tasks has traditionally been described as mostly nonstrategic. Recent findings indicate, however, that

The authors thank Beth Kurtz for her comments on an earlier version of this manuscript and Barbara Gollwitzer and Gabi Golling for their assistance in data collection. We are grateful to the staff and children of various kindergartens in Munich for their friendly cooperation. Requests for reprints should be addressed to Wolfgang Schneider. Max Planck Institute for Psychological Research, Leopoldstr. 24, 8000 München 40. West Germany. 
under certain task conditions preschoolers make deliberate efforts directed at the specific aim of remembering (Baker-Ward, Ornstein, \& Holden. 1984; Wellman, in press). Little is known, however, about young children's metacognitive understanding of the behaviors they display on memory tasks. Whereas in research with grade school children there is strong evidence in support of the hypothesis that knowledge about the requirements of a memory task and about the effectiveness of memory strategies is a necessary precondition for strategy use. metamemory-memory behavior relationships have rarely been studied in preschool children (see Schneider, 1985, for a review).

In a study on preschoolers" use and knowledge of organizational strategies in a sort-recall task, Sodian, Schneider, \& Perlmutter (1986) found that correlations among knowledge about the effectiveness of conceptual clustering. use of conceptual clustering at encoding and recall. and memory performance were significant in 6-year-olds but not in 4-year-olds. Although most 4-year-olds clearly organized the items conceptually at encoding and recall, this preference for conceptual clustering was not reflected in their subsequent ratings of different strategies. It would appear, then, that organizational behaviors are displayed by young children without a metacognitive understanding of the effectiveness of the strategies for memorization. Because only about $50 \%$ of the 4-year-olds, however. showed an acceptable level of consistency in their judgments in the metamemory interview, the apparent memory behavior-metamemory lag may simply have been due to a poor task understanding in 4-year-olds.

If young children possess a metacognitive understanding of the behaviors they display on memory tasks, this understanding should be clearer and more articulate the more natural a memory task is for the child. In quasinaturalistic studies with very young children memory-for-location tasks have been successfully used (e.g., DeLoache. 1980). Among other purposes these tasks have been employed to study the development of cueing strategies in young children. Cueing strategies seem to be used proficiently by very young children: Geis and Lange (1976) found that even 4-yearolds, when hiding pictures of people in houses. made some spontaneous use of the fact that the houses were marked with pictures of objects which were semantically related to the people's social roles le.g. . crownking). A similar cueing strategy could be prompted even in 3-year-olds (Ryan, Hegion, \& Flavell, 1970). Young children's use of cueing strategies seems to be dependent, however, on the strength of the semantic association between cue and target item: In a study by Gordon and Flavell (1977) 3 -year-olds placed cues appropriately only when cue and target were highly associated; Ritter, Kaprove. Fitch, \& Flavell (1973) found that young children did not use picture cues at retrieval when the cues were placed face down in front of them: in a task where there was no semantic relation between cues (paper clips) and target objects (cups where a 
candy was hidden) Ritter (1978) found that preschoolers used cues only after a series of prompts and that there were significant differences between younger and older preschoolers in the preparation, retention, and retrieval of a distinctive marker. These results suggest that young children's behavior in hide-and-seek tasks where there are strong semantic relations between cues and hidden objects may reflect an automatic tendency to put pictures with related objects rather than a deliberate, truly strategic attempt at remembering (see Ritter, 1978; Whittaker, McShane, \& Dunn, 1985).

One way to find out whether and to what extent young children employ retrieval cues in a deliberate attempt to aid prospective retrieval is to investigate their metacognitive understanding of retrieval cue utilization. During the last few years, several studies have been conducted to explore this issue. Fabricius and Wellman (1983) found that knowledge about effective and ineffective cuc locations develops only during the elementary school years. Beal (1985) showed, however, that while most of the criteria to make a cue informative were only understood by older children, 4and 5-year-olds were aware of some of the basic requirements for retrieval cues, for instance that the cue should be associated with the target item. and that an encounter with the cue is necessary for retrieval to occur.

To date. there has been only one study that directly explored the relationship between young children's cue utilization and their understanding of cue utilization (Whittaker et al., 1985). Whittaker et al. found that 3-year-olds could be induced to produce cueing behaviors without being aware of their usefulness for remembering. whereas 6-year-olds showed clear metacognitive awareness but sometimes failed to produce cueing behaviors spontaneously. The authors concluded that understanding of memory strategies may develop only as a by-product of their use. It should be noted, however, that Whittaker et al., 's youngest subjects rarely produced cueing behaviors spontaneously, but were induced to use cues by a number of prompts the experimenter gave. They may have produced cueing behaviors simply in an attempt to please the experimenter without understanding the rationale behind these behaviors. The relationship between memory behaviors and metacognitive understanding of these behaviors may be quite different with behaviors young children produce spontaneously.

The aim of the present study was to investigate the relationships among planful behaviors, metacognitive awareness of the functions of these behaviors, and memory performance in young children. For this purpose we used a task similar to Geis and Lange's (1976). for which spontaneous cue utilization has been reported even in 4-year-olds. Pictures of people were hidden in and retrieved from houses, half of which were marked with a picture of an object which was conceptually related to one of the people's social roles, while the other half were marked with pictures which were not related in a conventional way to the people. We explored 
whether and to which extent preschoolers made use of the semantic association between cues and targets and whether the use of related cues improved their performance on two memory tests (relocating the hidden pictures and free recall). In a task-related metamemory interview. we investigated whether the use of retrieval cues was accompanied by an awareness of the function of retrieval cues.

It is possible, of course, to also use semantically unrelated cues as memory aids by establishing idiosyncratic relations between cue and target. We tried to find out whether subjects were employing such a sophisticated elaboration strategy by asking them to give reasons for their hiding behaviors, that is, to tell why they had hidden a particular person in a particular house, after they had completed the hide-and-seek task.

Two experiments were conducted to investigate metamemory-memory behavior relationships on a memory-for-location task. In Experiment 1 the relationships among the use of retrieval cues, the knowledge about the usefulness of cues, and memory performance were studied in 4- and 6-year-old children. Geis and Lange (1976) found no age trends in the use and effectiveness of retrieval cues in their task over the elementary school years. Even their results for 4-year-olds appeared to be similar to those of the grade-school children. although the preschoolers were not tested in the same experiment. By comparing the performance of 4 and 6-year-olds, we attempted to investigate developmental trends in metamemory-memory relationships over the preschool years. Experiment 2 was conducted to gain more information on metamemory-memory relations in the younger age group (4- to 5-year-olds), adding a number of experimental controls to the procedure employed in Experiment 1.

\section{EXPERIMENT 1}

In Experiment 1 children's memory and metamemory were assessed in a two-trial memory-for-location task. A baseline trial in which the subject was presented with 10 unmarked houses in which the pictures of pcople were hidden preceded the experimental trial in which the houses were marked with picture cues, half of which were semantically highly associated to the people pictures while the other half were not. The differences in hiding times between the baseline and the experimental trial served as a first indicator of planfulness on the retrieval cue task.

A second, more direct indicator of planfulness at storage was children's use of the related picture cues at hiding. Whether subjects used an elaboration strategy to establish meaningful relationships between people pictures and "unrelated" cues could not be determined by behavioral indicators. Questioning young children about the reasons for their hiding behaviors may be problematic for a number of reasons: Children may. for instance, lack the verbal skills to give reasons for their hiding behaviors. 
or they may, on the other hand. be induced to "invent" post hoc justifications for arbitrary hiding. However, justifications seemed useful to gain a first insight into the rationale behind children's placements.

The memory-for-location task was followed by a short metamemory interview. Two items of this interview tested children's awareness of the critical features of the task. that is. of the difference between the baseline and experimental trials and of the superiority of related over unrelated cues. The other two items tested knowledge of other characteristics of a useful cue. that is, that it should be visible at the time of retrieval and that it should be unambiguous. Although the knowledge of these requirements is not crucial for adequate performance on the hide-and-seek task we used, these items were included to gain more information on preschoolers metacognitive understanding of cognitive cueing.

\section{Method}

Subjects. A total of 48 children from middle-class backgrounds participated in this study. 24 in each age group. An equal number of boys and girls was included at each age level. The 4-year-olds ranged from 4 (years), 0 (months) to 4.11 (mean age 4,5 ), the 6 -year-olds ranged from 5,5 to 6,11 (mean age 6,2). All subjects attended kindergartens in Munich. West Germany.

Materials. Ten small wooden houses $(14.0 \mathrm{~cm}$ long. $9.2 \mathrm{~cm}$ wide, and $5.0 \mathrm{~cm}$ high) were used. The roofs of the houses could be opened and shut like lids of boxes. Each house had a small magnetic sticker on its front side to affix the picture cue. Two identical sets of people pictures $(12.0 \times 7.4-\mathrm{cm}$ colored drawings $)$ were prepared, including a doctor, farmer, policeman. soccer player, sailor, ballet dancer, king, photographer, cook, and clown. Ten small $(4 \times 4 \mathrm{~cm})$ colored drawings served as picture cues; five of these were functionally related to the people pictures (syringe. tractor, police car, ball, and ship) and the other five were not directly related to the people pictures (comb. letter. key. flower, and lamp)

Procedure. Subjects were tested individually in a quiet room in their kindergarten. A "baseline task" and the true experimental task were administered to each subject followed by a metamemory interview.

In the baseline task the houses did not bear the picture cues. The 10 houses were placed in front of the subject in a semicircle. The 10 people pictures were arranged in front of the child in random order, that is, ordered differently for the various subjects. After the child had named each of the persons $s$ /he was told to hide each of the persons in one of the houses. The child was also told that $s /$ he would be asked to find the persons later. The experimenter recorded the time the subject took to hide the persons. After the children had completed hiding the persons, 
the experimenter asked them to name the colors on a color chart for 20 sec. The experimenter then picked up the duplicate set of people pictures and placed them in random order in front of the subjects with the instruction to lead each person to the house where her "twin brother or sister" was hidden (the term "twin" was explained if necessary). The time the subject took to locate the twins was recorded. After the child had put each "twin" in front of a house, the experimenter opened the houses and counted the number of correct hide-match correspondences.

This baseline trial was followed by the experimental trial. The experimenter labeled the houses with the picture cues in a predetermined random order. After the child had named the objects depicted on the cues, $s$ /he was asked to hide each of the persons again in a way that it would be easy for him or her to find the person later. Precautions were taken that, for each child, the arrangement of persons differed from that on the baseline trial. Again. the hiding time was recorded. Hiding was followed by a distractor task (counting for $20 \mathrm{sec}$ ). After this the "twins" had to be matched with their hidden partners as in the baseline trial. The time spent on the matching task and the number of hide-match correspondences were recorded.

After the houses were opened. the five houses with unrelated cues and two houses with related cues (i.e.. with a police car and a soccer ball) were pointed out and the subject was asked whether s/he could give any reason why $\mathrm{s} /$ he had hidden a person in this particular house. Questions concerning the two related cues were always asked last. The people pictures were then removed from the table while the houses with the picture cues remained there. The child was then asked to tell the experimenter the names of all the persons who had been in the game.

The free recall task was followed by a short metamemory interview. Each subject was asked the following four questions:

(1) Presence of retrieval cues: "Which of the games I just played with you was easier: Was it easier to find the people in the first game when you could not see the pictures on the houses or was it easier to find them in the second game when there were pictures on the houses or was it the same both times? Why?"

(2) Visibility of a cue: The house with the ball and the soccer player were pointed out to the child: "This is the soccer player and this is his house (soccer player was placed in the house). Is it easier to find him when I place his house like this (picture cue facing the child) or is it easier to find him when I place his house like this (picture cue turned away from the child) or is it the same both times? Why?"

(3) Cue relatedness: The policeman, a house with the related cuc (police car), and a house with an unrelated cue (lamp) were pointed out to the child: "Is it easier to find the policeman when I put him in this house 
(unrelated cue) or when I put him in this house (related cue) or are they both the same? Why?"

(4) Informativeness vs. ambiguity of cues: A house with a picture of a syringe, two houses with identical pictures of a ship, a doctor, and a sailor were placed in front of the child. The doctor was hidden in the house with the injection needle, the sailor in one of the houses with a ship: "Is it easier to find the doctor or is it easier to find the sailor or are they both the same? Why?"

\section{Results}

In a preliminary analysis the data were examined for sex of subject. Sex was included in an analysis of variance with the other variables of interest to ensure that no interactions with sex were significant. Since no significant effects of sex were found, the data were collapsed across this variable in all subsequent analyses.

In presenting the results, we first focus on a comparison of subjects behavior and performance in the baseline trial and experimental trial. Results on planfulness at storage and retrieval in the experimental trial will be presented next. In the last section. the metamemory data and the relationships among metamemory, strategic behavior, and performance will be analyzed.

Relocation of people pictures. A repeated-measures analysis of variance with age group as the between-subjects factor and testing session as the within-subjects factor was conducted on the number of hide-match congruences, that is, the number of locations that the children remembered correctly. There was a significant effect of test session, $F(1,46)=68.27$. $p<.01$. Independently of age, more locations were correctly remembered at the second trial than at the baseline trial. In addition, the Age $\times$ Test session interaction proved significant. $F(1,46)=7.42, p<.05$. Post hoc Student-Newman-Keuls tests showed that, although the six-yearolds remembered more locations on both trials, only the difference obtained for the second trial was statistically significant. Means and standard deviations of the dependent variables are given in Table 1.

Hiding time. Mean number of seconds spent in hiding the person pictures was analyzed in a repeated-measures analysis of variance with age group as between-subject factor and testing session as within-subject factor. The main effect of testing session was significant, $F(1,46)=$ 29.82. $p<.01$. Children of both age groups spent significantly more time hiding the person pictures during the second trial than during the baseline trial (see Table 1: the respective means were $118.12 \mathrm{vs} .77 .67 \mathrm{sec}$ for the 4-year-olds and 118.54 vs. 61.04 for the 6-year-olds). This finding is in line with the results obtained by Geis and Lange (1976) who found that grade school children (first, third, and fifth graders) spent more time in hiding the pictures when the cues were available than when they were 
TABLE 1

Means and Standard Deviations (in Parentheses) of Relevant Dependent Variables as a Function of Age Grolip in Experiment 1

\begin{tabular}{|c|c|c|}
\hline \multirow[b]{2}{*}{ Dependent variable } & \multicolumn{2}{|c|}{ Age group } \\
\hline & 4-year-olds & 6-year-olds \\
\hline \multicolumn{3}{|l|}{ Hiding time (sec) } \\
\hline Trial 1 & $\begin{array}{c}77.67 \\
(27.30)\end{array}$ & $\begin{array}{l}61.04 \\
(22.89)\end{array}$ \\
\hline Trial 2 & $\begin{array}{l}118.12 \\
(41.02)\end{array}$ & $\begin{array}{l}118.54 \\
(70.67)\end{array}$ \\
\hline \multicolumn{3}{|l|}{ Locations remembered $(\max =10)$} \\
\hline Trial 1 & $\begin{array}{c}1.50 \\
(1.64)\end{array}$ & $\begin{array}{l}2.58 \\
(1.82)\end{array}$ \\
\hline Trial 2 & $\begin{array}{r}4.08 \\
(3.22)\end{array}$ & $\begin{array}{l}7.70 \\
(2.87)\end{array}$ \\
\hline Cue use at storage $(\max =5)$ & $\begin{array}{c}2.0 \\
(1.98)\end{array}$ & $\begin{array}{c}3.63 \\
(1.93)\end{array}$ \\
\hline Meaningful justifications for hiding $(\max =7)$ & $\begin{array}{r}1.54 \\
(2.80)\end{array}$ & $\begin{array}{c}4.33 \\
(2.58)\end{array}$ \\
\hline Free recall $(\max =10)$ & $\begin{array}{l}4.79 \\
(2.43)\end{array}$ & $\begin{array}{l}9.25 \\
11.391\end{array}$ \\
\hline Metamemory $(\max =8)$ & $\begin{array}{l}3.50 \\
(2.09)\end{array}$ & $\begin{array}{l}5.00 \\
12.02)\end{array}$ \\
\hline
\end{tabular}

absent. The data of the present study indicate that this is also true for preschool and kindergarten children.

Cue use at storage and its effects. The number of pictures appropriately hidden (i.e., hidden with their related cues) was taken as a measure of the subjects tendencies to prepare themselves for future retrieval by using the conceptual relationships inherent in the materials. The older children were more planful at storage than the younger children, $t(46)$ $=2.88, p<.05$. On the average, the 6-year-olds hid 3.63 out of the 5 possible person pictures with their related cues, whereas the 4-year-olds hid an average of 2.0 person pictures appropriately (see Table 1).

As a first indicator of the relationship between planful storage and subjects' performance on remembering the location of the hidden objects. Pearson correlation coefficients were calculated for each age group. The coefficients were .72 and .91 for 4-year-olds and 6-year-olds. respectively. As already noted, 6-year-olds recalled more locations of pictures correctly than 4-year-olds (7.70 vs. 4.08; see Table 1). The correlation coefficients suggest that planfulness at storage had a pronounced effect on remembering the correct locations of person pictures, even in 4-year-olds.

Sixteen 4-year-olds and 21 6-year-olds hid at least one person with her related cue. For these children, a 2 (age) $\times 2$ (way hidden) split-plot 
analysis of variance was performed on the proportion of locations remembered correctly. As in Geis and Lange's (1976) study, this analysis was done at the subject level. The way-hidden factor refers to whether pictures were hidden with their related cues or not. Hidings with unrelated cues included both people pictures for which there were no related cues and people pictures for which there was a related cue which was not used. Main effects of age and way-hidden were significant, $F(1,35)=$ $13.15, p<.01$, and $F(1,35)=10.82, p<.01$, respectively. Post hoc comparisons revealed that 6 -year-olds were significantly better than 4year-olds at relocating both pictures hidden with their related cues, $t(35)$ $=2.87, p<.01$, and pictures hidden with unrelated cues, $t(46)=3.70$. $p<.01$. The respective mean proportions of 4-year-olds' and 6-yearolds' correct relocations for pictures hidden with their related cues were .63 and .94; those for pictures hidden with unrelated cues were .37 and .71. While 6-year-olds were close to ceiling in relocating pictures which they had hidden with their related cues, they were also very good at relocating pictures hidden with unrelated cues.

A closer inspection of the data showed that this was due to older children's better memory for the location of those pictures for which there was no related cue available: Only eight 6-year-olds hid less than five pictures with their related cues. For these children, the mean proportion of pictures relocated for which there was a related cue available but not used was .34. In contrast, the proportion of pictures relocated for which there was no related cue available was .73 for 6 -year-olds and .41 for 4-year-olds, $t(46)=3.62, p<.01$.

One reason for the age trend observed in the unrelated cue category may be that older children built meaningful relations between people pictures and unrelated cues more often than younger children. An analysis of children's explanations of why they had hidden a particular person in a particular house showed that 6-year-olds gave significantly more meaningful justifications than 4-year-olds: A justification was considered meaningful whenever the child explicitly established a functional relationship between the cue and the person picture in question (e.g., "I put the dancer into the house with the comb on it because she needs to comb her hair very often," "the cook is in the house with the lamp because he needs light for cooking"). This included justifications where the child elaborated a whole story about the relationship between cue and target (e.g., "The clown is in the house with the letter, because he received a letter from the cook who invited him for dinner"). Meaningful justifications were given a score of 1 , whereas responses like "I just did it" or "I don't know" were scored as 0 . Thus, the maximum score possible was 7. Scoring of justifications was not difficult: the interrater reliability coefficient was .93 . The few conflicting cases were solved by discussion. Four-year-olds reached an average score of 1.54, whereas 6- 
year-olds' average score was $4.33(t(46)=3.59, p<.01)$. Most 4-yearolds could explain why they had hidden a person with its related cue. but did not give any meaningful explanations for the persons hidden with unrelated cues. Seven 4-year-olds and nineteen 6-year-olds gave explanations for hiding persons with unrelated cues in addition to two correct answers to the questions about the persons hidden with related cues.

An analysis of successful relocations of items for which there was no related cue available as a function of children's justifications for hiding showed that mean proportions of hide-match congruences were 43 for the 4-year-olds and .53 for the 6-year-olds when a meaningful justification was given. In contrast, proportions of relocation were .29 for 4-yearolds and .39 for 6 -year-olds when no meaningful justifications were given. The $t$ tests revealed that there were no significant differences in proportions of relocations between the two age groups if hide-match congruences were regarded as a function of children's justifications. It should be noted. however, that only 74 -year-olds but 19 6-year-olds gave at least one meaningful justification and were thus included in the analysis.

Free recall. As can be seen from Table 1, the 6-year-olds recalled considerably more person pictures after the completion of the experimental trial than the 4 -year-olds, $t(40)=7.80, p<.01$. On the average, the older subjects remembered more than nine out of ten items and thus operated close to ceiling. Free recall was closely related to planfulness at storage (i.e.. number of items hidden with their related cues) and the number of meaningful justifications given by the subjects, regardless of age. The Pearson correlations between planfulness at storage and recall were .62 and .70 for the 4 -year-olds and 6 -year-olds, respectively. The corresponding coefficients for the relationship between meaningful justifications and recall were .70 and .62 . All of these correlation coefficients were significant at the $p=.01$ level.

Metamemory. Each item on the metamemory interview wals given a score of 0 whenever the first question was answered incorrectly, a score of 1 whenever the first question was answered correctly. and a score of 2 whenever both the first question and the subsequent "why" question were answered correctly.

The numbers of subjects who reached a score of 2,1 , or 0 on each of the four questions are shown in Table 2.

Whereas $50 \%$ of the 4-year-olds gave a correct answer and justification to question 3 (meaningful relation between cue and target), only $13 \%$ of the 4-year-olds and $21 \%$ of the 6-year-olds reached a score of 2 on the ambiguity question. These findings are in line with Beal's (1985) results. Somewhat surprisingly, comparatively few 4-year-olds reached the maximum score of 2 on questions 1 (presence vs. absence of cues) and 2 (cue visibility); this may have been due to a lack of verbal skills. als many 4-year-olds simply justified their answer hy saying "because it is 
TABLE 2

Number of Subjects with a Score of 2 (Correct Answer and Justification), 1 (Correct Answer), and 0 in the Metamemory In'terview in Experiment I

\begin{tabular}{|c|c|c|c|c|c|c|}
\hline & & & $\mathrm{Ag}$ & & & \\
\hline & & 4 & & & 6 & \\
\hline Item & 2 & 1 & 0 & 2 & 1 & 0 \\
\hline Presence of cues & 4 & 8 & 12 & 12 & 5 & 7 \\
\hline Visibility & 9 & 9 & 6 & 15 & 7 & 2 \\
\hline Cue-relatedness & 12 & 6 & 6 & 20 & 2 & 2 \\
\hline Ambiguity & 3 & 5 & 16 & 5 & 2 & 17 \\
\hline
\end{tabular}

easier to remember"; these tautological justifications were not counted as correct. In sum, children's knowledge about the use of cues seems to increase over the preschool years in all but one of the areas investigated: 6-year-olds were not more knowledgeable about the requirement that a cue should be unambiguous to be effective than 4-year-olds.

Metamemory, memory behavior, and memory performance. A correlational analysis of the relationships among the four metamemory items, the two measures of memory behavior (i.e.. planfulness at storage and justifications for hiding the pictures), and the two memory performance measures (number of person pictures correctly relocated and amount of recall) showed that metamemory was closely related to memory behavior and memory performance in both age groups (see Table 3).

Obviously, the point-biserial correlation coefficients depicted in Table 3 are not only statistically significant but also substantial. By and large,

\section{TABLE 3}

Point-Biserial Corkelations among the Four Mftamemory Items and Various Mfasures of Memory Behavior and Pfrformancf., Separately for Each Age Group (Data for 6Year-Olds in Parentheses) in Experimfint 1

\begin{tabular}{|c|c|c|c|c|}
\hline $\begin{array}{l}\text { Metamemory } \\
\text { items }\end{array}$ & $\begin{array}{l}\text { Cue use } \\
\text { at storage }\end{array}$ & $\begin{array}{l}\text { Locations } \\
\text { remembered } \\
\text { correctly }\end{array}$ & Recall & $\begin{array}{c}\text { Meaningful } \\
\text { justifications }\end{array}$ \\
\hline \multirow[t]{2}{*}{ Presence of cues } & .69 & .84 & .68 & .59 \\
\hline & $(.49)$ & $(.66)$ & $(.50)$ & $(.59)$ \\
\hline \multirow[t]{2}{*}{ Visibility } & .58 & .59 & .50 & .44 \\
\hline & $(.60)$ & $(.76)$ & $(.53)$ & (.60) \\
\hline \multirow[t]{2}{*}{ Cue-relatedness } & .52 & .70 & .46 & .36 \\
\hline & $(.38)$ & (.56) & $(.53)$ & $(.40)$ \\
\hline \multirow[t]{2}{*}{ Ambiguity } & .43 & .59 & .61 & .41 \\
\hline & $(.33)$ & $(.60)$ & $(.60)$ & $(.44)$ \\
\hline
\end{tabular}

Note. All correlation coefficients were significant at the $p<.05$ level. 
the pattern of correlations was roughly comparable across the two age groups. It seems particularly impressive that there were already close relationships between metamemory and memory in 4-year-old children. Somewhat surprisingly, no developmental trends were found in this data. While this finding may be partly due to the restricted variation in the memory performance variables (e.g., recall) obtained for the 6-year-olds. this explanation does not hold for the memory behavior variables.

\section{Discussion}

Experiment 1 focused on the relationships among metamemory, memory behavior, and memory performance in preschool and kindergarten children. The results support the assumption that even very young children's planful behaviors in memory tasks are accompanied by some degree of conscious awareness of the usefulness of these behaviors. We found significant correlations between metamemory and memory behavior (i.e.. use of retrieval cues) and memory performance (i.e.. relocating hidden ohjects and free recall) in 4-year-olds. Given that these correlations were already substantial in this age group, the fact that no increase with age was found does not seem surprising.

On the other hand, the results of Experiment 1 indicate that there are several developmental trends in the use and understanding of cueing strategies over the preschool and kindergarten years. An important developmental trend seems to be the increasing ability to spontaneously establish meaningful relations between cues and targets which are not highly associated. The justifications children gave for their choices of hiding places showed that the majority of the 6-year-olds established a relationship between a substantial number of cues and targets in the "unrelated cue" category and that even some 4-year-olds could justify their choice of hiding places in a reasonable way. As this measure is highly dependent on verbal skills, 4-year-olds" tendency to form elaborations may have been underestimated. In any case, the number of explicit elaborations in 6-year-olds was surprisingly high, considering previous accounts of kindergartners' strategic behaviors on memory tasks. One might argue that the justifications for the choices of hiding places do not necessarily indicate that children used an elaboration strategy at the time of storage but that they might have invented post hoc justifications. By letting children choose whether they had had any special reason for hiding a picture at a particular place or whether they had "just put it there" we tried to reduce the pressure to give a reason even if there was none. The fact that the number of meaningful justifications given was highly intercorrelated with the success at relocating the pictures in both age groups suggests that this measure may serve as a first attempt to investigate elaboration strategies at storage.

Geis and Lange (1976) did not observe age trends in either cue use or 
cue effectiveness in grade school children. Our results indicate that during the preschool years children not only become more able and inclined to use cues but that their cue use also becomes more effective. One of the reasons for this increased effectiveness may be an increase in knowledge about the functions of retrieval cues. The metamemory data showed that while 4-year-olds understand that cues should be conceptually related to targets, 6-year-olds can give adequate reasons for the advantages of conceptual relations more often than 4-year-olds: moreover. they can give adequate reasons why it is better to have cues at all than to just try and find the items without the help of cues, and they know some of the requirements of cue placement. Given their restricted knowledge about cueing. 4-year-olds may rely less firmly on cues as memory aid even if they use them at storage. This may be the reason for their lower success in relocating items even if they were appropriately hidden.

However, even in 4-year-olds the use of experimenter-defined cues proved highly more effective for relocating the items than arbitrary placements. The metamemory data showed that about $50 \%$ of the 4-year-olds could give correct answers and justifications to the question about the superiority of semantically related over unrelated cues. Thus, the findings of Experiment 1 suggest that 4-yeat-olds have a basic understanding of cueing as a memory aid.

It should be noted, however, that the metamemory data as well as some of the memory data could be positively biased duc to the experimental design of Experiment 1. That is, the fact that the metamemory interview always came last could have resulted in reports representing after-thefact noticing that may be unrelated to metamemory as a precondition of strategy use. Similarly, the fact that the baseline trial always preceded the experimental trial could have contributed to improved memory performance in trial 2. Consequently, cue/no cue and trial effects were confounded in the analyses concerning retrieval cue effects.

Experiment 2 was primarily designed to determine the relative importance of order effects for the major conclusions from Experiment 1. and to replicate the finding that substantial correlations among measures of metamemory, memory behavior, and memory performance can be obtained in a memory-for-location task even in 4- to 5-year-old children.

\section{EXPERIMENT 2}

The procedure used in Experiment 1 was modified to include a number of additional experimental controls. In order to control for the possibility that young children simply report on their own previous behaviors in the metamemory interview without being aware of the usefulness of these behaviors for remembering the items, an additional experimental condition was included in which the metamemory interview preceded the memory task. The metamemory interview was restricted to task-related items, 
that is, it was redesigned to include two three-item scales, one testing children's knowledge about the usefulness of the presence of cues and the other one testing their knowledge about the superionity of semantically related over unrelated cues. Thus the relationship between children"s knowledge that a particular item should be hidden with its related rather than with an unrelated cue and their actual hiding of this item could be assessed for a subset of three items. According to the metamemory hypothesis (Flavell. 1971), knowledge about a memory strategy should be a precondition for its use. If this is true for 4-year-olds' knowledge and use of retrieval cues. children should only use semantically related retrieval cues if they know that a semantically related cue is more useful than an unrelated one in aiding future retrieval.

In order to estimate to what extent children's improved performance on the second trial of the memory task was due to the use of retrieval cues versus increased familiarity with the items, the experimental trial was presented without baseline testing to a subgroup of subjects in Experiment 2.

An additional purpose of Experiment 2 was to study the relative effectiveness of experimenter-defined vs. child-defined cues. In Experiment 1, even some of the younger children gave meaningful justifications for their use of cues which, by experimenter-defined criteria, were not related meaningfully to the target items. It is quite possible that children's criteriat may deviate from adults" also in those cases where. by experimenterdefined criteria. there is a strong semantic association between cue and target: that is, children who do not use "semantically related" cues may be ignoring them hecause they have established strong semantic relationships between cues and targets which deviate from the experimenterdefined ones. These self-established relationships may, however, be as effective in aiding retrieval as reliance on conventional semantic relattionships. In order to investigate children`s own criteria in hiding the people pictures, subjects were asked to justify their placements of all 10 people pictures subsequent to the memory lask. Thus it was possible to assess children's success at relocating the items as a function of appropriate hiding. where appropriateness was defined as the use of a hiding place which was meaningfully justified thy positing a functional relationship between a cue and a target) by each individual child.

\section{Method}

Subjects. Forty-eight children. 26 boys and 22 girls from middle-class backgrounds participated in Experiment 2. Their mean age was 4.6 (range $3,10$ to 5,3$)$.

Design and procedure. Half the subjects received the memory task first and the metamemory interview second (memory-metamemory condition), and the other half received the two tasks in the reverse order 
(metamemory-memory condition). Within each of these two groups, 16 subjects received both the baseline and the experimental trial, whereas 8 subjects received the experimental trial only.

Under the memory-metamemory condition the procedure was the same as in Experiment 1 except for the following alterations: After the people pictures had been relocated in the experimental trial, children were asked to give reasons for the placement of all 10 people pictures. The metamemory interview consisted of six questions: In the first, fourth, and sixth question, children were presented with one people picture and two houses, one of which carried a cue, whereas the other one did not. The pairs of people pictures and cues were: Policeman-policecar, cook-lamp, and soccer player-soccer ball. Children were asked whether it was easier to find the person if she was hidden in the house with a cue or in the house without a cue, or whether this was equally easy in both cases, and why (Cue-presence scale). In the second, third, and fifth question, children were presented with a people picture and two houses, one of which carried a cue which was semantically related to the people picture and one of which carried a semantically unrelated cue. The triplets of people pictures and cues were: Doctor-letter-syringe, farmer-tractor-ball, and sailor-key-ship. Children were asked whether it was easier to find the person if she was hidden in the house with the related cue, or if she was hidden in the house with the unrelated cue, or whether it was equally easy in both cases and why (Cur-telatedness scalc).

In the metamemory-memory condition children were first introduced to the task by showing them the 10 houses. some of which carried cues, while others did not. The experimenter explained that in the game people had to be hidden in the houses and to be relocated later on. She then showed the people pictures to the children and asked them to name the persons on the pictures. Then she pointed out the cues to the children and let them name the objects which were depicted on the cues. She then said that she wanted to ask the children about the best way to hide the people in this game in order to find them later on. After this all houses except for two were removed and the metamemory questions were asked. After the metamemory interview was completed the experimenter proceeded with the memory task in the same way as in the memory-metamemory condition.

Results

Preliminary analyses showed no significant effects of sex of subject on any of the dependent variables. Thus, the data were collapsed across this variable for further analyses.

In presenting the results, we first analyze the effects of the experimental manipulations (position of metamemory interview and presence vs. absence of baseline trial). The effects of cue use at storage on memory performance 
TABLE 4

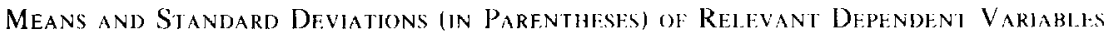
as a Function of Expraimfintal. Conidition in Experimfint 2

Experimental condition

Dependent variable

Hiding time (sec)

Trial 1

Trial 2

Locations remembered $(\max =10)$

Trial 1

Trial 2

Cue use at storage $(\max =5)$

Meaningful justifications for hiding (max - I())

Free recall $(\max =10)$

Metamemory (max

12)
Memorymetamemory

With Without With Without baseline baseline baseline baseline

\subsection{1}

$(19.52)$

114.06

(42.47)

(2)

101.6 .3

$(18.49\}$

102.06

109.75

(47.16)

$(40.32)$

$144.12)$

(4)

$\begin{array}{lccc}1.25 & - & 2.13 & - \\ (1.18) & & (1.89) & \\ 4.56 & 3.00 & 4.25 & 3.75 \\ (3.71) & (2.73) & (2.84) & 12.38) \\ 2.50 & 1.75 & 1.88 & 1.63 \\ (2.03) & (1.90) & (1.99) & 11.841 \\ 3.56 & 2.13 & 3.50 & 2.63 \\ (3.14) & (2.42) & (3.46) & 13.25) \\ 6.06 & 4.25 & 6.13 & 3.50 \\ (3.97) & (2.05) & (2.45) & (2.98) \\ 7.06 & 6.38 & 7.25 & 0.63 \\ (3.09) & (2.88) & (3.911 & (3.46)\end{array}$

will be presented next. followed by the metamemory data, and the results of the analysis of interrelations among metamemory. strategic behavior. and memory performance.

Effects of the experimental manipulations. Table 4 shows the means and standard deviations for the dependent variables hiding time. number of locations remembered, number of pictures hidden with their related cues. number of meaningful justifications for hiding, number of pictures recalled, and metamemory (sum score) under each of the four experimental conditions, memory-metamemory condition with baseline (1). memorymetamemory condition wihout baseline (2), metamemory-memory condition with baseline (3), and metamemory-memory condition without baseline (4). The metamemory sum score was computed by adding the sumscores of each of the metamemory subscales. As in Experiment 1. each item on the metamemory interview was given a score of 2 (correct answer and justification), 1 (correct answer), or (). Thus the maximum score on each of the subscales was 6 . 
A 2 (position of metamemory interview) by 2 (presence vs. absence of baseline trial) analysis of variance revealed no significant effects of the position of the metamemory interview on any of the dependent variables, nor were there any interactions between this variable and the presence vs. absence of the baseline task. Specifically, there were no significant effects of the position of the metamemory interview on the metamemory sum score or on either of the sum scores of the two metamemory subscales. Thus, the data were collapsed across the "position of metamemory interview" variable for further analysis.

Planned comparisons of the results for the groups with and without baseline task showed no significant effects of the presence of the baseline task on hiding time at trial 2 , on the number of pictures relocated correctly on trial 2, on the number of pictures hidden with their related cues, on the number of meaningful justifications for hiding, and on the metamemory data. However, children who received the baseline trial recalled significantly more pictures than children who did not, $t(46)=2.40, p<.05$. The difference in number of items recalled was largely due to a higher recall rate for those pictures for which there were no related cues available (average numbers of 1.38 vs. 2.63 out of five pictures correctly recalled in the groups without and with baseline trial, respectively). $(46)=2.45$. $p<.05$. For the pictures with related cues the difference only approached significance (2.5 vs. 3.47 out of five pictures recalled), $t(46)=1.93, p$ $<.10$.

A comparison of Table 4 with Table 1 shows that the average number of pictures correctly relocated in Experiment 2 was comparable to that obtained in Experiment 1 for 4-year-olds. In free recall, the group who received both the baseline and the experimental trial was slightly better than the same-aged children in Experiment 1.

As in Experiment 1. children who received the baseline trial relocated significantly more items on the second trial than on the first, $t(15)=$ 3.63, $p<.01$, for experimental condition 1 (see Table 4), and $t(15)=$ $3.11, p<.01$, for experimental condition 3. Although children who received both the baseline and experimental trials (conditions 1 and 3) were slightly superior to children who received only the experimental trial (conditions 2 and 4 ) on the number of pictures relocated on trial 2 , this difference was not statistically significant. Thus the improvement on the second trial can be mainly attributed to the presence of retrieval cues, not to the repeated exposure to the items.

Those children who received the baseline trial spent significantly more time in hiding the pictures on the second trial than on the first, $t(15)=$ 4.94, $p<.001$, for experimental condition 1 , and $t(15)=3.91, p<.001$ for experimental condition 3. Again. as Table 4 shows, this difference was due to the presence of cues at the second trial, not to the repeated 
exposure to the task which might have induced a more reflective attitude on the second trial.

Cue use at storage and its effects. On the average, children hid 2.02 out of 5 people pictures with their related cues, that is, appropriately by experimenter-defined criteria. This average number of correct placements is almost the same as the one observed for 4-year-olds in Experiment 1. As in Experiment 1, the use of related cues at storage was highly correlated with the number of items correctly relocated, $r=.67$. Thirtyone children hid at least one person picture with its related cue. For these children, the mean proportion of items correctly relocated was .65 . In contrast, the mean proportion of items correctly relocated if hidden with unrelated cues was $.41, t(30)=3.88, p<.01$. These and the following analyses were done on the subject level in the same way as described in Experiment 1.

Similar effects of cue use at storage on remembering the locations of hidden items were found when appropriateness at hiding was defined by children's own criteria: When asked to justify their hidings. children gave an average number of 2.0 meaningful justifications for their hidings of pictures for which there was a related cue available, and an average number of 1.15 meaningful justifications in those cases where there was no related cue available. In the related-cue category there was a high correspondence between children's and experimenter's criteria, $\phi=.78$. Only 13\% of those subjects who used an experimenter-defined related cue could not give a meaningful justification for their cue-use, and only $8 \%$ of those children who did not hide one of people pictures for which there was a related cue available with the experimenter-defined cue at storage, gave a meaningful justification for their deviating choice of a hiding place. The number of meaningful justifications given and success at relocating the items were highly intercorrelated, $r=80$. Thirty-one children gave at least 1 (out of 10) meaningful justification for their choices of hiding places: two of these children gave 10 such justifications. For the remaining 29 children the mean proportions of items successfully relocated were .68 when the hiding was meaningfully justified and .40 when it was not meaningfully justified, $t(28)=4.51, p<.01$. Thus the mean proportions of hide-match congruences for appropriate and inappropriate hidings when appropriateness was defined by children s own criteria were almost identical to those found when appropriateness was defined by experimenter's criteria. A 2 (appropriateness) by 2 (children 's vs. experimenter's criteria) split plot analysis of variance on the proportion of hide-match congruences revealed a significant main effect of appropriateness. $F(1,24)=14.17, p<.001$. No significant main effect of the factor "children's or experimenter"s criteria" was found, nor wats there a significant interaction.

As there was a close correspondence between experimenter's and 
children's criteria in hiding those items for which related cues were available, and as the number of meaningful relationships created for the hiding of pictures with unrelated cues was small, it is not surprising that the proportion of hide-match congruences were equally high when appropriateness was defined by children's as when it was defined by experimenter's criteria. However, when only those hidings were considered which were justified meaningfully by children, a comparison of the mean proportions of hide-match congruences of those items which were hidden with their related cues, and those which were hidden with an unrelated cue. showed that hiding with a predefined related cue was more effective than creating one's own relationship between cue and target. $t(28)=$ $3.32 . p<.01$ (the mean proportions were .62 and .26).

Fre' recall. As already noted, the number of items recalled subsequent to the hide-and-seek task was affected by the number of trials in the task: Children who received the baseline and the experimental trials recalled significantly more pictures than children who received only the experimental trial. However. repeated exposure to the items was ohviously not the only factor which affected free recall: Cue use at storage was not only intercorrelated with the number of locations remembered but also with the number of items recalled. Pearson correlations between appropriate hiding and free recall were $r=.61$ when appropriateness was defined by experimenter's criteria and $r=.65$ when appropriateness was defined by children's uwn criteriat.

Metamemory. A sum score was computed for each of the two scales of the metamemory interview as described above. On the scale assessing children's awareness of the usefulness of the presence of cues fitems 1 . 4 , and 6) the average score was 3.06, on the scale assessing the awareness of the usefulness of cue relatedness (items 2. 3. and 5) the average score was 3.87 . Table 5 shows the number of subjects who received a score of 2. 1. or 0 on each of the six interview items. With the exception of item 4 . at least $50 \%$ of the subjects answered and justified correctly each

TABI.F. 5

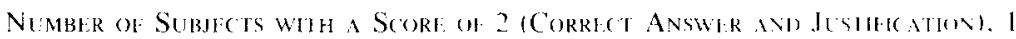
(CORRI:C A ANSWFR), (RR () IN IHA. MEIAMIMORY INTERVHW IN FXPHRIMENI 2

Metamemory

scale

Item

Scorc

$\begin{array}{lrrrr} & & & \\ \text { Presence of cues } & 1 & 23 & 6 & 19 \\ & 4 & 5 & 12 & 31 \\ \text { Relatedness of cules } & 6 & 34 & 5 & 4 \\ & 2 & 25 & 6 & 16 \\ & 3 & 34 & 6 & 17\end{array}$


of the metamemory items. Item 4 differed from items 1 and 6 in that a house with an umelated cue was compared to a house without a cue. Thus, correct answers and justifications on item 4 depended on understanding that a meaningful relationship could be formed between a person picture (the cook) and an unrelated cue (the lamp) and that therefore a house with an unrelated cue was still superior to a house with no cue at all. In sum, results of the metamemory interview are comparable with the answers to the cue-relatedness question in Experiment 1 (see Table 3), and slightly better than the results on the cue presence and cue visibility items in Experiment 1 for 4-year-olds.

Metamemory, memory behavior, and memory performance. Table $\mathrm{s}$ shows the point-biserial correlations among each of the six items of the metamemory interview, the two measures of planfulness at storage (number of items hidden appropriately by experimenter-defined criteria and number of meaningful justifications for hidings as a measure of children 's own criteria), and the two measures of memory performance (number of items correctly relocated and free recall).

A comparison of Table 6 with Table 3 (Experiment 1) shows that correlations between metamemory items and each of the measures of memory behavior and performance, although significant, were slightly lower than in Experiment I. Imperfect correlations between metamemory and memory behavior may be due either to a high number of subjects who know about the usefulness of cues but do not use them or to a high number of subjects who use a cueing strategy without heing aware of its function in aiding retrieval. The latter case is incompatible with the prediction that metamemory should be a precondition for strategy use. An inspection of the relationship between children's answers on the three metamemory items of the cur-relatedness scale and their hiding hehaviors for each of the three pictures showed that for an average of $60 \%$ of the

TABILO

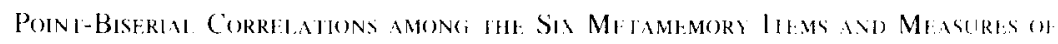

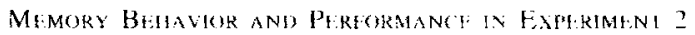

\section{Metamemory} scale

Presence of ews

$\begin{array}{ll}4 \\ \text { Relatedness of etues } & 6 \\ 3 \\ 3\end{array}$

$$
\begin{aligned}
& \text { Cue use } \\
& \text { Item at storage }
\end{aligned}
$$$$
\begin{array}{r}
\text { Locatio } \\
\text { remember } \\
.38 \\
.43 \\
.47 \\
.48 \\
.50 \\
44
\end{array}
$$$$
\begin{array}{r}
.36 \\
.25 \\
.32 \\
30 \\
.35 \\
.24
\end{array}
$$

Note. All correlation coefficients were significant at the $p$
Meaningful justifications

$\begin{array}{cc}\text { Frec } & \text { Meaningful } \\ \text { recall } & \text { justification } \\ & \\ 410 & .40 \\ 38 & .50 \\ .43 & .35 \\ .34 & .50 \\ .47 & .38 \\ .47 & .37\end{array}$


subjects the answers on the metamemory interview corresponded with their behaviors in the memory task, that is, they either reached a score of 0 or 1 on the interview and hid the picture inappropriately or they gained a score of 2 and hid the picture appropriately. An average of $30 \%$ of the children received a score of 2 on the metamemory question but hid the corresponding picture inappropriately. The remaining $10 \%$ scored 0 or 1 on the metamemory interview but showed appropriate hiding behaviors; the exact figures for this last category were 5. 7, and 3 subjects on items 2, 3, and 5, respectively. Thus few subjects hid a picture appropriately without knowing which hiding place made it easier to relocate the picture. Separate crosstabulations of metamemory items and hiding behaviors for the two experimental conditions (memory-metamemory and metamemory-memory) revealed no significant differences between the two groups.

In order to be qualified as "strategic" a hiding behavior has to occur with some regularity. In order to test the prediction made by the metamemory hypothesis that strategy use does not occur without corresponding metamnemonic knowledge, we defined those children as "strategic" who hid at least two of the three items in question with their related cues. A child was considered to possess metamnemonic knowledge about appropriate hiding when he or she gave correct answers and justifications on at least two of the three interview questions of the cucrelatedness scalc. Under this definition. only 3 (out of 48 ) children behaved strategically at hiding without knowing about the hiding strategy. In order to test whether these data support the prediction that metamemory is a precondition for strategy use, a prediction analysis (Hildebrand, Laing. \& Rosenthal, 1977) was performed on the $2 \times 2$ table created by crossing metamemory with strategy use. The hypothesis that without accurate metamemory strategy use does not occur was confirmed, $z=2.03, p<$ .05 .

\section{Discussion}

In Experiment 2 the main results of Experiment 1 were replicated. Most importantly, it was shown that young children's answers on the metamemory interview did not merely reflect post hoc reports on the behaviors they had just displayed on the memory task. Rather, children showed an equal level of metamnemonic knowledge when the metamemory interview preceded as when it followed the memory task. This suggests that even without prior experience concerning the specific memory task used in this study, 4- to 5-year-olds possessed some declarative knowledge about the usefulness of cues for prospective retrieval and about the superiority of semantically related over unrelated cues.

As in Experiment 1, the use of experimenter-defined cues proved to 
be highly effective for aiding retrieval even in 4-year-olds. Moreover. it was shown that almost $90 \%$ of those children who used experimenterdefined related cues at hiding, were able to justify their choice of hiding places by referring to the functional relationship between cue and target. This suggests that 4- to 5-year-old children did not merely react automatically to the presence of highly associated cues but were able to reflect on their choice of cues. In those cases in which semantically related cues were available, children's criteria for appropriate hidings rarely differed from experimenter's. When children created their own relationships between target items and unrelated cues, they were less successful in relocating the items than when they relied on preestablished semantic relationships. This is in line with findings on the early development of organizational strategies (Sodian et al. . 1986) which indicate that even 4-year-olds are able to use semantic relationships among items to facilitate remembering and that the use of semantic relationships is effective even in this age group.

Additional evidence for the effectiveness of a cueing strategy in 4- 10 5 -year-olds was provided by the results of the experimental condition in which no baseline trial preceded the experimental trial. Measures of menory behavior and of memory performance at relocating the items were not significantly affected by the omission of the baseline trial. Thus, the presence of retrieval cues, not the repeated exposure to the items to be remembered, scems to have been the major cause for children's increased success at relocating the items on the second trial of the twotrial task. Children who received both the baseline and the experimental trials did recall significantly more items than children who received the experimental trial only. This seems plausible, as. on the two-trial task. children saw the same stimuli on two rccasions which should facilitate recall of the stimulus names, but not necessarily of their changing locations.

Experiment 2 provided further support for the assumption that metamnemonic knowledge about cueing is functionally related to the use of a cueing strategy even in 4- to 5-year-olds. Cue use at storage was significantly intercorrelated with knowledge about the functions of ctue presence and semantic relatedness of cues to targets. Moreover, the data tentatively support the prediction made by the "metamemory hypothesis" that metamnemonic knowledge is a precondition for strategy use. Whereas a sizeable proportion of subjects who knew about the function of semantically related cues in aiding retrieval did not employ a cueing strategy at storage, very few children showed the opposite pattern. This was true independently of whether the metamemory interview preceded or followed the memory task. Thus. metamemory seems to be a necessary (but not sufficient) condition for strategy use in a cognitive-cueing task in 4 - to 5-year-old children. 


\section{GENERAL DISCUSSION}

Previous studies have shown that preschoolers do not use retrieval cues spontaneously if there is no strong semantic association between cue and target, or if this relation is not very salient in the situation (e.g. Gordon \& Flavell, 1977; Ritter, 1978). This has led to the interpretation that preschoolers may react automatically to highly associated cues without any awareness of the value of cues in aiding prospective retrieval. The present results indicate that this account of preschoolers' understanding of cognitive cueing may not be entirely correct. Even 4-year-olds were able to justify their use of semantically related retrieval cues by referring to the functional relationship between cue and target. In a task-related metamemory interview, about two-thirds of the 4-year-old children knew that it was easier to relocate hidden items if retrieval cues were available than if they were not, and that it was easier to relocate items if they were hidden with semantically related than with unrelated cues. About half of the 4-year-olds not only knew that suitable retrieval cues facilitate remembering but could also give adequate reasons for this facilitating effect. Of course, young children's reasoning about mnemonic processes cannot be expected to be very explicit. Thus, children typically referred to the semantic relationship between cue and target when asked why it was easier to remember an item hidden with its related than with an unrelated cue. It is not possible to determine on this basis whether and to what extent children understood the reasons why semantic relationships between cues and target items can be used to facilitate remembering. that is. whether they possess an intuitive theory about the epistemic effects of cognitive cueing. However, the fact that the majority of the 4- and 5-year-old subjects in Experiment 2 were able to give correct answers and justifications to the metamemory questions even if these questions preceded the memory task, suggests that very young children possess a stable and statable piece of basic knowledge about cognitive cueing which is not merely a by-product of an experience with a specific memory task.

The present results support the assumption that young children's metacognitive knowledge about the use of retrieval cues, limited as it may be, is functionally related to their use of retrieval cues in a memory task. In both experiments. measures of metamemory and memory behavior were shown to be substantially intercorrelated even in 4-year-olds. Close relationships between metamemory and memory behavior have, to our knowledge, not been demonstrated previously in this age group. An inspection of the kind of relationships between metamemory and memory behavior tentatively supported the assumption that knowledge about the effectiveness of a cueing strategy was a necessary but not sufficient 
precondition for its use. This is in line with findings for older children (see Schneider, 1985). Further research would have to show whether similar metamemory-memory relationships can be demonstrated in preschoolers on different memory tasks.

In addition to demonstrating relationships between metamemory and memory behavior. the present study highlighted some developmental trends in children's use and understanding of retrieval cues between the ages of 4 and 6 years. Both the use and the effectiveness of cues increased over this age period. Results of Experiment 1 indicate that one of the reasons for this increase in effectiveness may be an increase in knowledge about the functions of retrieval cues. A comparison of the findings for 4- and 6-year-olds suggests that the development of young children's understanding of cueing as a memory aid may proceed along the following lines: At first children realize that cues can be used as a source of information at retrieval if they are closely associated with the target item. In a second step. they seem to learn that cues can be actively manipulated both spatially (e.g., Whittaker et al., 1985) and conceptually by using an elaboration strategy.

In line with previous studies (Ackerman. 1982; Beal. 1985: Fabricius \& Wellman, 1983) our results indicate that even at the end of the kindergarten years, children still have a lot to learn about the use of retrieval cues. Even 6-year-olds did not know about one of the most important requirements to make a cue informative, that is, that it should not be ambiguous. As some justifications showed, they rather seemed to think "the more identical reminders the hetter." This indicates that cues as a means to communicate a message to oneself are not fully understood by kindergartners. A third developmental step in the understanding of cognitive cueing may thus be the insight that cues can also be misleading and that one has to be carefil to make them truly informative.

\section{REFERENCES}

Ackerman. B. (1982). Retrieval variability: The inelficient use of retrieval cues by young children. Jommal of Exporimental Child Psyloleger, 33, 413-428.

Baker-Ward, L... Onstein, P. A. \& Holden, D. J. (1984). The expression of memorization in early childhood. Journal of Experimental (hild Pruchology, 37, 555-575.

Beal. C. R. (1985). Development of knowledge about the use of cues to aid prospective retrieval. Child Development, 56, 6.31-642.

Del oache, J. S. (1980). Naturalistic studies of memory for object location in very young ehildren. In M. Perlmutter (Ed.). Children's memory. San Francisco: Jossey-Bass.

Fatbricius, W. V.. \& Wellman, H. M. (1983). Children's understanding of retrieval cue utilization. Derelopmental Psychology, 19, 15-21

Flavell. J. H. (1971). First discussant's comments: What is memory development the development of"? Human Do'velopme'nt. 14, 272-278.

Geis, M. F., \& I.ange, G. (1976). Children's cue utilization in a memory for location task. Chiid De'lolopment, 47, 759-766.

Gordon. F. R., \& Flavell, J. H. (1977). The development of intuitions aboul cognitive cueing. Child Doretopment, 48, 1027-1033. 
Hildebrand. D. K., Laing, J. D., \& Rosenthal. H. (1977). Prediction andysis of crossclassifications. New York: Wiley.

Kreutzer, M. A., Leonard. C. \& Flavell, J. H. (1975). An interview of children's knowledge about memory. Monographs of the Society for Research in Child Dovelopment. 40. (1. Serial 159).

Ritter. K. (1978). The development of knowledge of an external retrieval cue strategy. Child Derelopment, 49, 1227-1230.

Ritter, K., Kaprove, B. H., Fitch, J. B., \& Flavell, J. H. (1973). The development of retrieval strategies in young children. Cognitive Psychology, 5, 310-321.

Ryan, S. M., Hegion, A. G., \& Flavell, J. H. (1970). Nonverbal mnemonic mediation in preschool children. Child Dovelopment, 41, 539-550.

Schneider, W. (1985). Developmental trends in the metamemory-memory behavior relationship: An integrative review. In D. L. Forrest-Pressley, G. E. McKinnon, \& T. G. Waller (Eds.), Cognition, metacosnition, and human performance (Vol. 1). New York: Academic Press.

Sodian. B.. Schneider, W. \& Perlmutter, M. (1986). Recall, clustering, and metamemory in young children. Jourmal of Experimental Child Psychology, 41, 395-410.

Wellman, H. M. (1977). Preschoolers understanding of memory relevant variables. (hild Development, 48, 1720-1723.

Wellman. H. M. (in press). The early development of memory strategies. In F. F. Weinert \& M. Perlmutter (Eds.). Memory development: Universal changes and individual differences. Hillsdale. NJ: Erlbaum.

Whittaker. S. J. (1986). Eliminating alternatives: Preschool children's use of indirect memory cues. British Joumal of Derelopmental Psuchologx, 4, 199-207.

Whittaker, S.. MeShane, J., \& Dunn. D. (1985). The development of cueing strategies in young children. British Joumal of Developmental Psychology, 3, 153-161. 